\title{
The Future of Architectural Design in the Post-Digital Era
}

\author{
AIMEE BUCCELLATO \\ University of Notre Dame \\ HOLLY FERGUSON \\ University of Notre Dame \\ CHARLES F. VARDEMAN, II \\ University of Notre Dame
}

Our presentation at the 2016 AIA Intersections Symposium described a multi-disciplinary research agenda that ponders where architecture, as a discipline and a practice, sits with respect to the age of ubiquitous data. That presentation and the synopsis that follows is focused on our on-going development of novel tools and frameworks to advance decision support for building design and construction in this context. Apropos to the workshop theme, Innovative Technologies in Design and Delivery, our work is motivated by emerging technologies in computational and data science that may revolutionize the way the built environment is conceived and produced and, consequently, what that means for the Future of Design in a Post-Digital Era.

\section{BACKGROUND AND MOTIVATION:}

First, to establish what we mean by Post-Digital Era. Several years ago, pioneering computer scientist Jim Gray offered that we are moving into a wholly new era in scientific discovery, what he termed to be The Fourth Paradigm (Hey). Whereas previous eras of scientific discovery were marked by advances in observation, theory, and then observation and theory supported by computational analysis, we have entered yet another revolution - The Fourth Paradigm - wherein altogether new ways of conducting research through Data analysis are being discovered. The Fourth Paradigm, which exists beyond the Computational Era, marks newfound ability to access large amounts of heterogeneous data in order to make discoveries that would not be possible in a single view of the data from a single data set. Furthermore, Fourth Paradigm technologies transform our ability to make decisions using that data; in ways that would be virtually impossible previously. Unlike preceding paradigms in discovery and research, which were marked by advances in observation and the generation and use of data, The Fourth Paradigm signifies our ability to comprehend data in completely new and useful ways [Figure 1].

\section{WHAT DOES THIS MEAN?:}

Today, we have access to data from around the world and we have technologies that allow us to gather this information into manageable, semantically-compatible formats such that the data can be made useful in an architectural design process. Where data is needed, missing, inconsistent, or incomplete, we have technologies to help us "fill in the blanks". Our design decisions can be influenced by myriad design tools and analysis approaches because of the increased interoperability afforded by modern technologies. And, perhaps most critically, because of these new technologies, we can begin to incorporate data from outside the immediate domain of architecture into the decision-making process; meaning, we can use data from domains that directly affect our design choices and design outcomes, but was previously incompatible with tools and processes without manual and unreliable human intervention.

\section{ARCHITECTURE, BIG DATA, AND A POTENTIALLY TRANSFORMATIVE APPROACH}

Given current methods and approaches for incorporating building design information and data into a holistic design process - generating, storing, and communicating via BIM - the focus of our research is on the challenges and areas of weakness in the state-of-the-art, and the intersection of architecture with non-domain-specific methods, tools, and frameworks that already exist - and that architecture could better exploit - in the design and execution of buildings. Among them, concepts with origins in Big Data that have the potential to help architects, among many other disciplines, "harness information in (new and) novel ways (in order) to produce useful insights or goods and services of significant value." (Cukier). The concepts behind the Big Data revolution are tightly tethered to another fundamental, yet potentially revolutionary idea: that all things that are discoverable are also linked (Barabasi). So, in addition to achieving better access to and utility of data, our understanding of networks and the relationships between things may very 


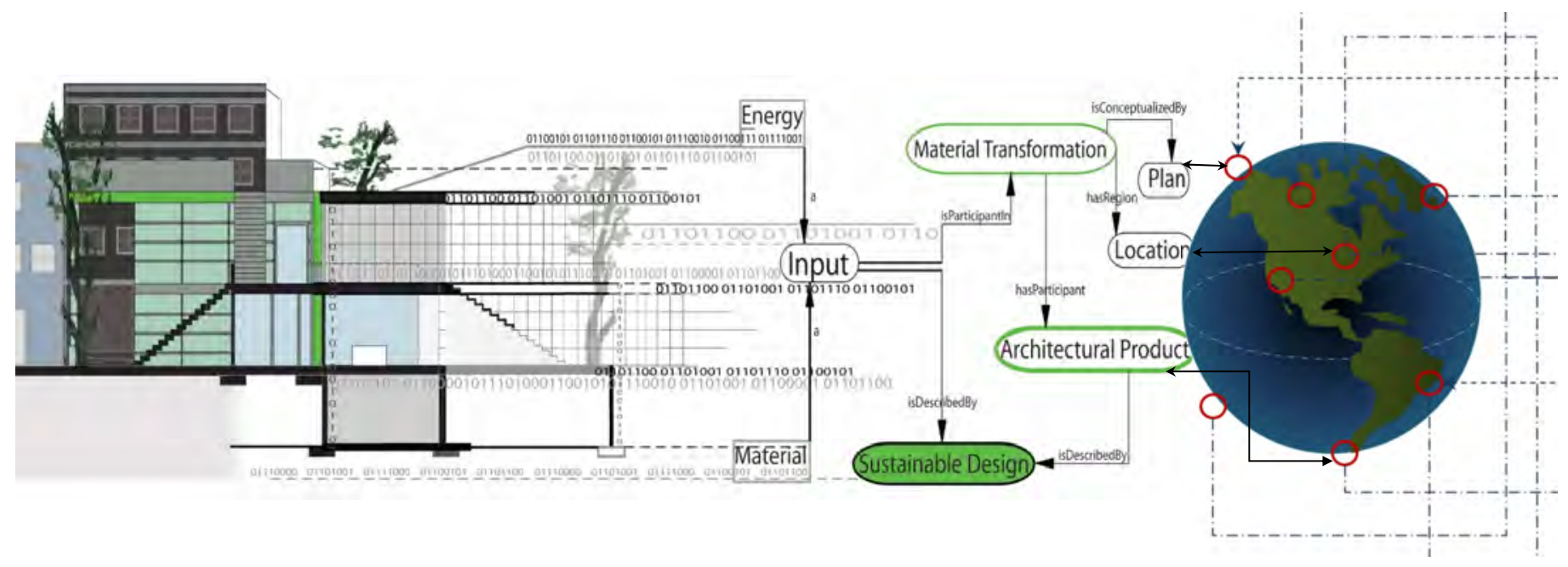

Figure 1. Development of Design from Base Material Observations to Computational Analysis to Modern Data Accessibility/Us

well transform the way we approach design, from the design of businesses, to vaccines, to buildings, to cities [Figure 2].

How, practically, will this transformation occur? How will we gain the necessary understanding? What can we possibly do with all of this data? Nearly every discipline is grappling with these questions. Our research is interested in determining how tools and concepts being ushered in by the Era of Big Data might be useful to and influential in the practice of architecture, along the following general themes:

First, how can we, the architecture community, more effectively use data and metadata to drive innovation in design and project delivery? Although recognized by industry, the ability to rapidly derive alternate, informationrich views of building models is touted by BIM applications as "critical for making quantitatively-informed design decisions" (Levy), and yet, we cannot always determine the origin of the source data or even the mathematical models behind the analysis and simulation conducted in these tools. Which means architects must not only understand the outputs of prevailing tools, but also how the models behind those tools work and generate insight, and, importantly, according to what data. Because ultimately, regardless of the interface, transparency, and interoperability, "good quality data is essential for architects to make more intelligent choices about how they design all projects". (Levy)

Next, how can we meaningfully advance collaborative practices in architecture in the age of ubiquitous data, information-rich drawings, and smart buildings? And, finally, what might happen if we are able to harness the knowledge of our broader community to grow and sustain new networks of information? For example, advanced computational models and methods that will help us explore the vast amount of building information and data that already exists and that is being generated, daily, by industry, by architects and allied experts, and by the buildings themselves.

In response, our research ponders the following topics and questions:

1. Understanding Structured Data and how our architecture, like others, can benefit from the generation, curation, and use of structured data in practice
2. Why is data access hard? And how does data access (and reliability) influence the practice of architecture, the decisions that architects make, and the impact of the buildings that we create?

3. Once we can achieve better access to data and more reliable data, what more powerful ways exist for harnessing it and using it? Can we utilize pattern-based approaches, like other disciplines?

4. Once more reliable data can be more efficiently and effectively accessed, how might advanced computational tools further support the architect in decision-making?

When thinking about answers to those questions, the kinds of methods and tools that we are developing fall into three main areas:

1. Novel multi-criteria decision support tools that

2. Use and leverage web-based strategies to streamline the creation and discovery of building information and design data, and

3. Enhanced decision support via rules engines and machine learning

\section{HOW DID WE GET “HERE”? DATA KNOWLEDGE EVOLUTION}

Several years ago, while pursuing a very specific question related to material property research, we discovered large gaps in both tools and data for accurately evaluating and comparing the broader impacts of the way we make and operate buildings, particularly with respect to the usefulness of these evaluation tools to the average student and practitioner and the availability of source data. Over the course of this inquiry, we discovered that the data we use - and that is used by prevailing design and analysis tools - is largely siloed and non-localized. Data existing in fragments and pieces, of varying quality and accessibility. And, yet, in spite of the fidelity of the data and the tools - or lack thereof - today's design and analysis tools - and the data they use - have a substantial influence over design decision-making. And this is the data that already exists -- some of it in databases, some open, some proprietary. What of the data being generated each day, by researchers of buildings, building materials, and the buildings themselves? 
We began to ask ourselves and, eventually, our colleagues across and allied to our discipline (Buccellato):

1. How do we currently access and building design information and data?

2. What are the barriers to it -- the data?

3. What data is missing?

4. How do we tap into the explicit and tacit knowledge of the built environment, data that's embedded and available, but NOT yet accessible, reliable, and usable?

\section{GIVEN ALL OF THESE CHALLENGES, HOW DO WE SOLVE THEM?}

1. How do we accelerate access to the data that we have and the data that we need?

2. What kind of model frameworks exist for data and knowledge acquisition, discovery, and sharing?

How are other disciplines approaching - and advancing - in the age of ubiquitous data? And what can WE learn from them? How do WE similarly tap into the potential in Big Data?

Although this is a non-trivial task, gaining broader access to building design data and related information presents myriad new challenges as well as timely opportunities to influence the future conception and execution of the built environment. So, how do we do it? Everyone could remain focused on building more robust tools and individual applications, simulation models, and databases, etc., but the broader data challenge would remain. Our strategy is to make the data smarter, make that data accessible and then create frameworks that use smart data, which will ultimately make everyone's applications smarter.

\section{NOW, WHERE DO WE “GO"? INTERDISCIPLINARY OPPORTUNITY:}

These are big challenges and therefore we, the architecture and allied community, need help. This is where we turn to experts in Big Data, Knowledge Engineering, and Decision Theory to expand our current understanding and ability to effectively harness data and information in a data-enabled design

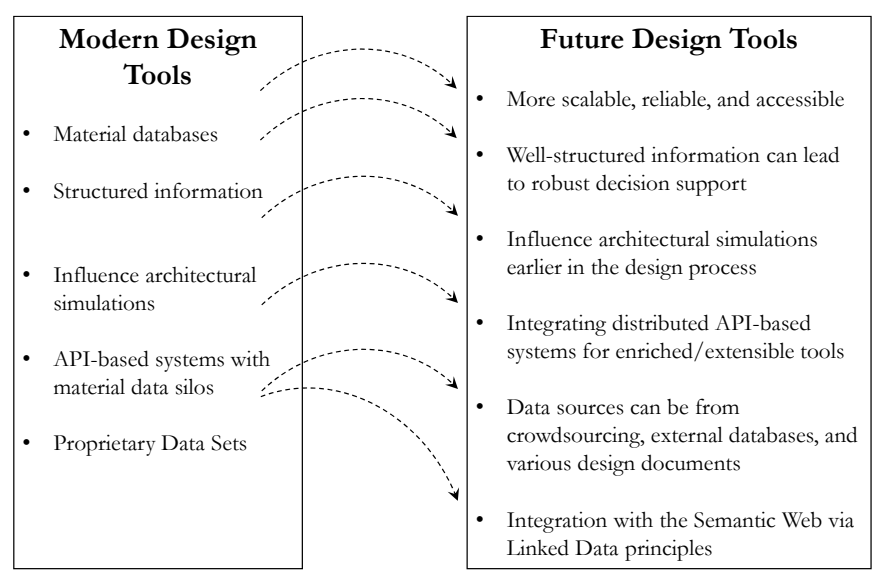

Figure 2. Comparison of Data in Modern Design Tools versus Future Design Tools

"A major paradigm shifts introduced by the
Semantic Web is to focus on the creation of
smart data instead of smart applications.
The rationale behind this shift is the insight
that smart data will make future
applications more (re)usable, flexible, and
robust, while smarter applications fail to
improve data along the same dimensions..."

Source: Krzysztof Janowicz, Frank van Harmelen, James A. Hendler, and Pascal Hitzler. "Why the Data Train Needs Semantic Rails." Al Magazine, 2014. http://corescholar.libraries. wright.edu/cse/169/.

process. Fundamental concepts that are changing the face of many dataaware and data-dependent disciplines that can also be used to mitigate the (data) challenges we face in architecture. These concepts, some of which are described below, include the Semantic Web ${ }^{1}$ (which introduces relationships to concepts as opposed to just definitions), pattern languages (which are used to computationally construct relationships and context between ideas and data), and specific ways that we are exploring the intersection of architecture and A.I. - or artificial intelligence - and the potential for architects to meaningfully leverage Al methods and tools in their work.

\section{COMPUTATIONAL MODELS AND METHODS TO EXPLORE BIG DATA: THE SEMANTIC WEB AND STRUCTURED INFORMATION}

This idea of making "smart data" instead of smart applications coincides with a major paradigm shift introduced by the Semantic Web. The World Wide Web (WWW) "invented" by Tim Berners-Lee was intended to be a web of connected data, not simply a web of connected documents; what most web users are familiar with in the form of hyperlinks and hypertext. In BernersLee's vision (Tim Berners-Lee), the web is an ecosystem of smart agents that can process smart data directly without human intervention. The WWW standards organization, the World Wide Web Consortium (W3C), has already developed a set of technologies and standards that enable this vision including graph representations of data (RDF) ${ }^{2}$ and standards for encoding formal logic in RDF, like OWL. ${ }^{3}$

These tools are powerful because they allow the formal representation of a human conceptualization in a structure that is understandable by machines. This is called an ontology. However, in order for an ontology to function and support a human-posed question, the data must be formatted a certain way (or be "query-able"). Linked Data is a set of principles that have been developed in the Computer Science fields to guide the publication of data on the World Wide Web to meet this formatting style. It is intended to accelerate and enable, really, the adoption of Semantic Web technologies, like the use of ontologies. Some of the rationale behind this shift is "... the insight that smart data will make future applications more reusable, flexible, and robust, leaving smarter applications to fail to improve data along the same dimensions" ( $K$. Janowicz). The primary thrust being: most modern tools, design-centered or otherwise, do not (yet) integrate how human beings think, work, or find useful information in their daily lives, let alone enable us to connect various types of data for simultaneous consideration and use. 


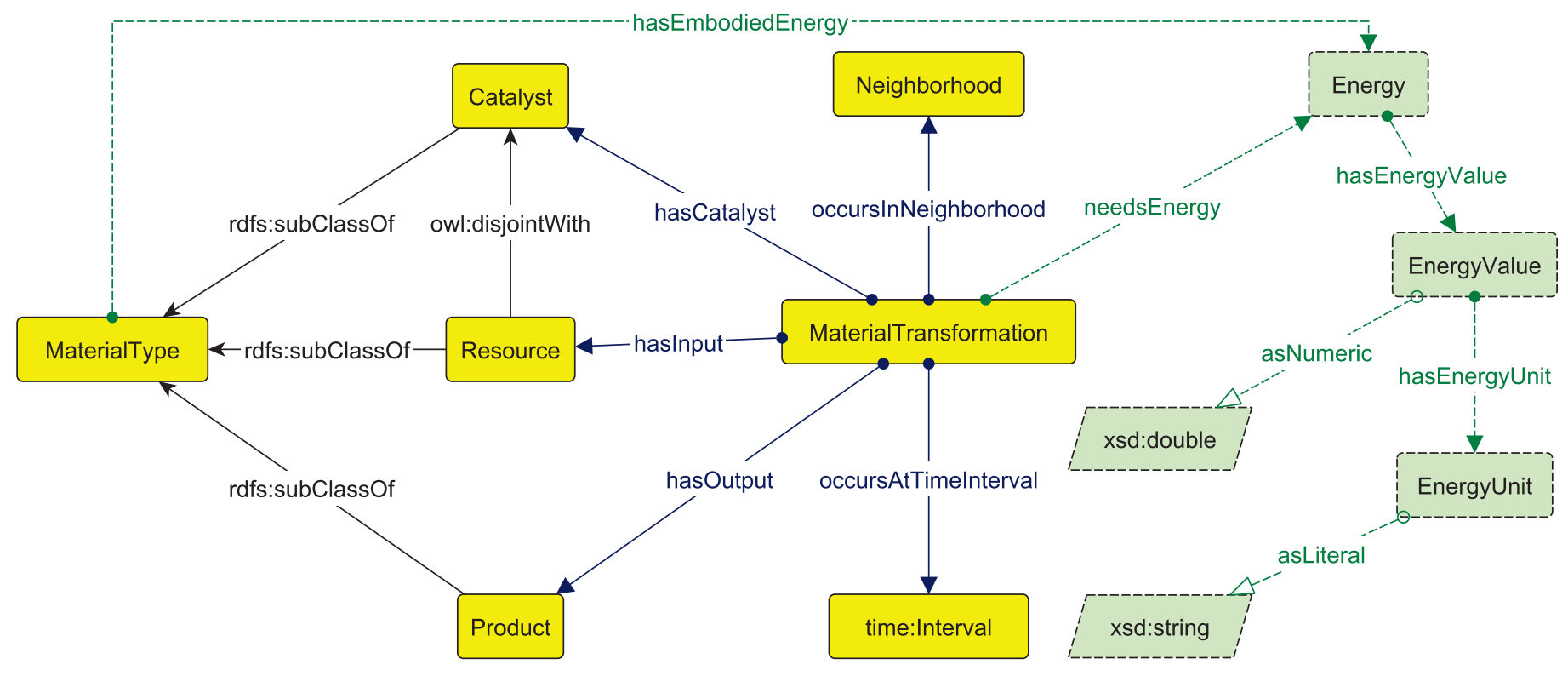

Figure 3. Example Data Patern: Material Transformation Pattern (To read more about these please visit: http://ontologydesignpatterns.org/wiki/ Submissions:ContentOPs)

If properly structured, Semantic Web technologies could transform the way that architects access building design information and data and, in turn, architects could exploit the assistance of machine agents in decision support, including the potential to use and manipulate distributed knowledge bases called Knowledge Graphs. A knowledge graph structure provides the basis for contextual information. An example of this is the additional information - dates, history, related events - that are returned in many Google searches of the WWW. ${ }^{4}$ If Google can provide useful, contextual information about a simple term lookup, imagine the opportunities for harnessing information about something as complex as the elements of building design. Our efforts are particularly focused on ways that these techniques and technologies could enhance and expand the data used during architectural analysis and thus advance our capacity to make sustainable and resilient design choices. A similar structure is used in

LinkedIn and Facebook, which is a type of Al used to determine suggested acquaintances. In lieu of the author/user expending all of the effort of searching, the smart agent connects pertinent information related to the data. Importantly, the decision to be connected is not made for us, but all of the information available is presented for human consumption and human action.

\section{LINKED OPEN DATA AND ONTOLOGY DESIGN PATTERNS}

What does it mean for data to be structured in a re-useable way that machines can understand within the Semantic Web? This process is essential to integrating these technologies with architectural design. The interoperable structures that make this all possible are called Ontology Design Patterns and are a type of Linked Data that not only connect data but also establish relationships between different data elements with formal logics.
A pattern-based approach of re-usable solutions makes it possible draw equalities and mappings between all of the different sets of data that we may encounter and use in modern tools.

For example, imagine that you would like to capture the motion or trajectory of a building material from raw material to installation at a construction site. You may be a construction manager or an architect concerned about the transportation impact of materials on embodied carbon. How would you describe the trajectory in a way that a computer could understand? Today, there are reusable patterns that allow us to track and record locationspecific, GPS, and other information that can be queried during analyses that track a "Semantic Trajectory" (Y. Hu) through space and time. For our more specific use-case, we have constructed a new pattern called a "Material Transformation" pattern [Figure 3] (Vardeman II) that "tells" a machine that something has changed identity in, say, a manufacturing process. In this pattern, a set of material inputs and outputs exist to the transformation and something must change between the inputs and outputs for a transformation to exist. This logic is enforced though machine readable axioms expressed in formal logic. Together, these two patterns, the existing Semantic Trajectory pattern and our new, Material Transformation pattern, enable a computer to understand how a building material moves from place to place and how to identify if a material has changed identity. The influence of these patterns on the collection of life-cycle inventory data, for example, could be significant.

\section{LINKED OPEN DATA AIDING DECISION SUPPORT}

Beyond the ability to structure data in an interoperable way to make it more easily discoverable and accessible, there are opportunities to computationally-support the analysis and use of information in new and potentially transformative ways. Current methods of analysis in architecture typically involve techniques that consider the information available compared with the preferences of the user (one example of this is the selection of preferred building materials or preferred manufacturers). In the realm of computer science, Decision Support techniques and technologies are developed to assist 


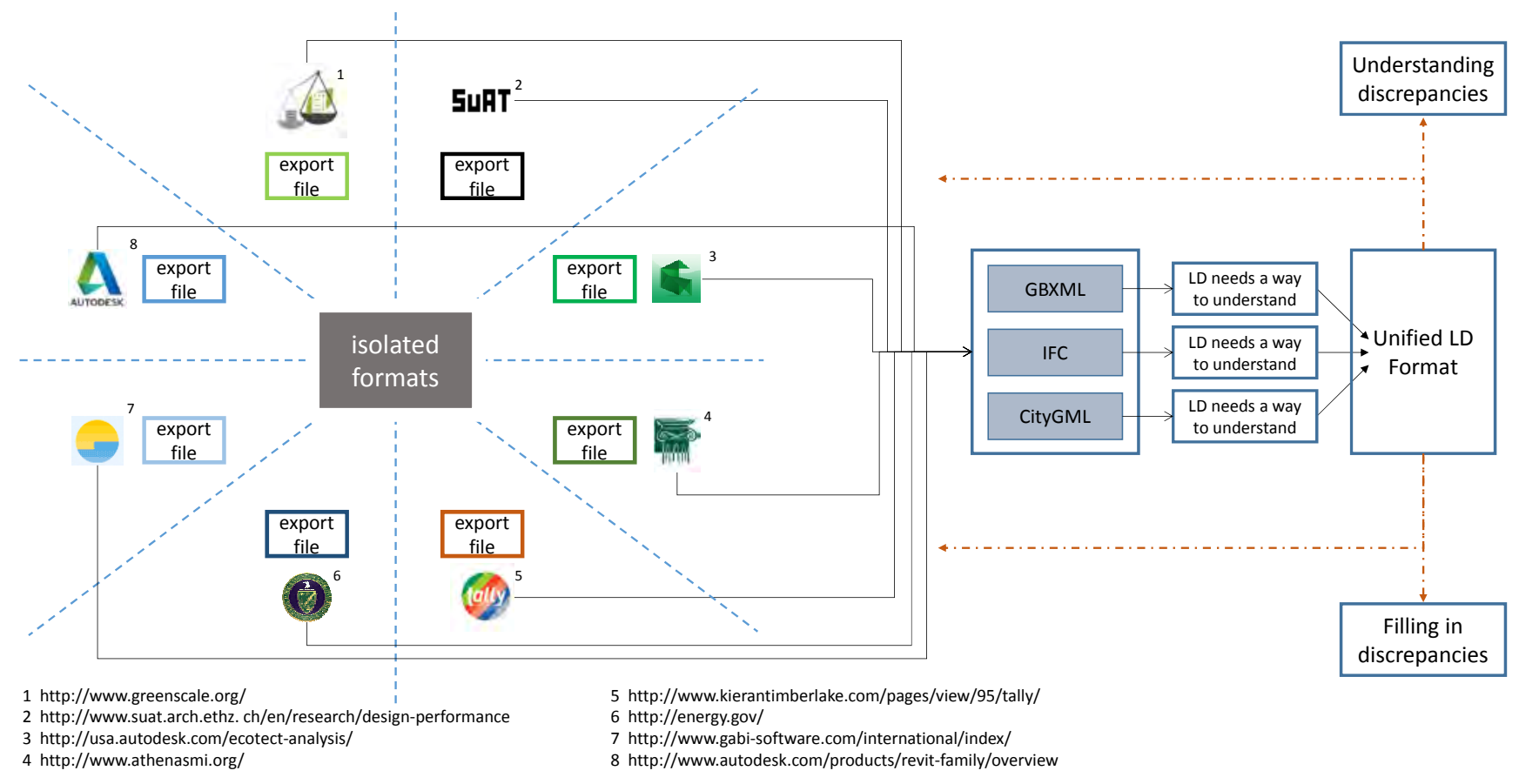

Figure 4. Extracting and Formatting Data that is Semantic Web Compatible

users in processing information, which is becoming increasingly essential in the Era of Big Data, in order to process the immense amount of information available or even to be able to ask our modern, multidisciplinary questions.

A foundational component for Decision Support now and in the future is smarter data, made possible by standards called Linked Data. With properly structured Linked Data, Decision Support can be orders of magnitude more accurate and comprehensive than what is currently used in individual design tools. For example, smart data-enabled Decision Support in building design could mean the ability to compare anticipated building energy use with embodied energy/ carbon for the entire lifespan of the building, competitive trade-off analysis of those choices, including degradation and replacement costs, and resilience-based concerns, including climate, hazard risk, and so on.

This level of analysis is clearly needed in the building industry, though it requires data and processing capabilities beyond what our current tools can perform. However, Semantic Web technologies can be combined with distributed cloud based data platforms, technologies which do already exist, in order to enable users to access remotely-located resources for Decision Support. The Cloud, when combined with Linked Data, already has powerful enough processing capabilities to advance Decision Support in the manner we are suggesting, all of which is needed in order to more fully support and enable data-aware architectural design. Meanwhile, it is important to acknowledge that Decision Support is a major part of our everyday lives, in the form of our smart phones and machine agents that already assist in controlling parts of the build environment, such as in smart homes. At minimum, shouldn't these existing technologies be informed with the most robust and complete data?

\section{ARTIFICIAL INTELLIGENCE: BENEFITING THE CREATIVE PROCESS}

Artificial Intelligence, or the ability of machines to exhibit intelligent behavior can benefit the human-centered, creative design process - as opposed to replacing it. If machines such as Watson 5 can "learn" to play competitive chess and comprehend trends in recipes and preferred flavor combinations in order to propose new, improved cooking ideas, then perhaps it is not too far-fetched to suggest that we could use the same tools to learn something useful about trends in building construction and performance that would provide insight for the design of new buildings. Thomas Malone, founding director of MIT's Center for Collective Intelligence, said that the "future lies in building systems that can best leverage the capabilities of humans and computers. A growing body of research is finding that answers gleaned from a combination of humans and computers are more accurate than those generated by either group alone."

Instead of hindering creativity, better data processing mechanisms can conserve design time previously spent manually sourcing and aligning data, leaving more time for actual creative design pursuits. Additionally, many of the simulations used for resilience calculations are able to utilize real-time and sensor-based data. The efficient and effective collection of this type of information for use in the creative process is not typically feasible without computational intervention without unreasonably burdening the creative process. Instead, data platforms using Semantic Web technologies can make sense of sensor data, for example, and draw conclusions computationally that will support architects with data analysis that is too complex to be done by humans in an efficient manner.

\section{CONCEPTS APPLIED: OUR CURRENT MULTIDISCIPLINARY RESEARCH PROJECTS AND FUTURE WORK}

As we assess and align our research with normative design processes and workflows, we design data patterns to extract information from building design models and filter it into a common and unifying format whose intelligence can be expanded as our Linked Data Platform (LDP) infrastructure grows. LDP is already a W3C standard and the patterns themselves are also already Linked Data. Therefore, data can be used in different simulations and serialized 
into a variety of formats. By virtue of using Linked Data-compatible formats, these technologies virtually eliminate the need to create "patch" solutions, in the form of individual tools or translators, for translating data between file formats. Communications within the LDP are designed specifically to promote the exchange of structured data and translation mechanisms and meanwhile provide passageways for the automated searching of additional data on the web to fill in the missing data, automatically correct the inconsistent data, and place information where data is otherwise incomplete. Additionally, these tools can be expanded in a "build-as-you-go" manner.

In prevailing 3-D modelling programs, BIM is what a computer uses to describe and understand what a particular design is. To use building information of this kind in a LDP environment means developing methods to automatically provide context between different data types, and as an extension, make BIM and related tools more interoperable. In other words we work on ways to translate data for use in several simulation types without the necessity of manual intervention, which is useful for improving predictive modelling, and for tracking data provenance information. This part of our research focuses on building linked-data compatible translation methods between the industry common data formats: IFC, GBXML, and CityGML, so that we can test and expand the capability of our data models to more effectively harness data and enable greater insight during the design process [Figure 4].

The development of these tools and technologies is extremely prescient and timely for the domains of architecture and engineering and the creation of the built environment, as the influence of our design decisions on the environment and human health are acknowledged to be significant. When we think back to the very earliest practitioners and theorists on architecture, there were a relative few types of materials suitable for the construction of buildings. Simply those, as Vitruvius observed, containing or made by the "primordial substances": earth, air, fire, water. Not so today: when compared to the sheer number of materials and methods that can be used to construct a building; and the growing expectations on architects and engineers to predict how their design decisions related to the combination of those many materials and methods will ultimately perform when constructed.

Ultimately, buildings are more complicated than ever before and we must design and make them faster. Owners, their representatives, banks, and building operators ask design teams to predict how those buildings can be expected to perform - on time, over time - in terms of energy consumption, long term durability of systems and assemblies, under normal conditions of degradation and even resilience against failure due to natural or man-made hazard. We are generating more data and information about our buildings and by our buildings - than ever before. So, there are challenges surrounding data-enabled design, even in its current state. Foremost among them:

1. Where is the data? And how much can we move to the open?

2. Can we get businesses and people to share their data and information for the common good?

3. If the will is there, for sharing, what are the mechanics?

4. Of the barriers to adoption of data-enabled practices - authorship, intellectual property, data quality, data validation - which are the biggest threats?
When advancing to the frontier of discovery, we need tools to prepare us to perform once we get there. We are not suggesting that these tools or methods - these cognitive agents -- will replace the human agent in design. What we are suggesting is the Future of Design in the Post-Digital Era lies in tools and cyberinfrastructure that will advance design and practice, enabling, as the legendary computer scientist Steve Jobs suggested, the machines to do the mundane, while empowering people to do the extraordinary. ${ }^{7}$

\section{ACKNOWLEDGEMENTS:}

We would like to thank the University of Notre Dame, the Notre Dame Center for Research Computing, Office of Research, and Center for Sustainable Energy. We would also like to thank the National Science Foundation under grant CMMI 1537652:"A Green Resilience Framework to Support the Design of Sustainable Buildings Under Multiple Hazards."

\section{ENDNOTES}

1. https://www.w3.org/standards/semanticweb/

2. https://www.w3.org/TR/rdf11-concepts/

3. https://www.w3.org/TR/owl2-primer/

4. https://googleblog.blogspot.com/2012/05/introducing-knowledge-graph-things-not. html

5. http://www.ibm.com/watson/

6. https://www.technologyreview.com/s/519831/

new-answer-from-ibms-watson-a-recipe-for-swiss-thai-fusion-quiche/

7. https://www.youtube.com/watch?v=ob_GX50Za6c

\section{REFERENCES:}

Barabasi, Albert-laszlo. Linked: How Everything Is Connected to Everything Else and What It Means for Business, Science, and Everyday Life. Basic Books, 2014.

Buccellato, A. P, et al. "Sustainability Data Community Forum." Chicago, 18-19 July 2013. Workshop.

Cukier, Viktor Mayer-Schönberger and Kenneth. Big Data: A Revolution That Will Transform How We Live, Work, and Think. Eamon Dolan/Mariner Books, 2014.

Hey, Tony. The Fourth Paradigm: Data-Intensive Scientific Discovery. Vol. 1. Microsoft Research, 2009.

K. Janowicz, F. van Harmelen, J. A. Hendler, and P. Hitzler. "'Why the Data Train Needs Semantic Rails"." Al Magazine 2016.

Tim Berners-Lee, James Hendler, and Ora Lassila. "'The Semantic Web"." Scientific American 2001: 28-37.

Vardeman II, C. F., Krishnadi, A. A., Cheatham, M., Janowicz, K., Ferguson, H., Hitzler, P., and Buccellato, A. P. "An Ontology Design Pattern and Its Use Case for Modeling Material Transformation." Semantic Web (2016).

Y. Hu, K. Janowicz, D. Carral, S. Scheider, W. Kuhn, G. Berg-Cross, P. Hitzler, M. Dean and D. Kolas. "A Geo-ontology Design Pattern for Semantic Trajectories." T. Tenbrink, J. Stell, A. Galton, Z. Woods, Eds. Spatial Information Theory. Springer International Publishing, 2013. 438-456. 